\title{
Preliminary comparison of neuropsychological performance in patients with non-small-cell lung cancer treated with chemotherapy or targeted therapy
}

This article was published in the following Dove Medical Press journal: Neuropsychiatric Disease and Treatment

\author{
Hsiu-Ling Kangl,* \\ Vincent Chin-Hung \\ Chen ${ }^{2,3, *}$ \\ Wei-Lin Hung ${ }^{4}$ \\ Han-Pin Hsiao ${ }^{2}$ \\ Wei-Han Wang ${ }^{4,5}$ \\ 'Department of Nursing, Chang \\ Gung Memorial Hospital, Chiayi, \\ Taiwan; '2Department of Psychiatry, \\ Chang Gung Medical Foundation, \\ Chiayi Chang Gung Memorial \\ Hospital, Taiwan; ${ }^{3}$ School of Medicine, \\ Chang Gung University, Taoyuan, \\ Taiwan; ${ }^{4}$ Department of Psychology, \\ Chung Shan Medical University, \\ Taichung, Taiwan; ${ }^{5}$ Room of Clinical \\ Neuropsychology, Department of \\ Neurology, Chung Shan Medical \\ University Hospital, Taichung, Taiwan \\ *These authors contributed equally \\ to this work
}

Purpose: This cross-sectional pilot study aimed to compare the effects of chemotherapy and targeted therapy on neuropsychological performance and psychiatric symptoms in patients with non-small-cell lung cancer (NSCLC).

Patients and methods: A total of 113 patients with NSCLC were recruited. According to their type of cancer treatment, the patients were classified into chemotherapy $(n=40)$, targeted therapy ( $n=33)$, and untreated control $(n=40)$ groups. All participants completed five objective tests measuring various domains of cognitive function, a subjective cognitive functioning scale (Functional Assessment of Cancer Therapy-Cognitive Function; FACT-cog), and the Hospital Anxiety and Depression Scale (HADS) either within 6 months after diagnosis (for the untreated group) or about 18 months after treatment.

Results: Overall, there were no significant intergroup differences in the proportions of patients with abnormal cognitive performance and psychiatric disturbances. Among the untreated NSCLC patients, $35 \%$ had impaired performance in at least one cognitive domain, and a comparable finding $(30 \%-35 \%)$ was made for the other two treatment groups. The proportion of patients with impaired psychomotor speed was the highest (10\%-15\%) across various cognitive domains. Moreover, a significant proportion of NSCLC patients (15\%-20\%) exhibited HADS-defined anxiety and depression disorder. Finally, significant correlations were found between FACT-cog total scores and the HADS Depression subscale across all three groups.

Conclusion: This study demonstrated that 1) a substantial proportion of NSCLC patients exhibited cognitive impairments (especially regarding psychomotor speed) and psychiatric disturbances; 2) no significant differences were observed among the three patient groups for any subjective or objective measure of cognitive deficit; and 3) perceived cognitive impairment was significantly associated with depression or anxiety. Prompt treatment of psychiatric disorders to minimize their impact is therefore recommended.

Keywords: non-small-cell lung cancer, neuropsychological performance, psychomotor speed, anxiety, depression

\section{Introduction}

Lung cancer has a high mortality rate worldwide and is also one of the leading causes of cancer-related deaths in Taiwan, accounting for $\sim 9,000$ deaths annually or $20 \%$ of all cancer-related mortalities. ${ }^{1}$

Chemotherapy is a type of cancer treatment that uses chemical agents to destroy all dividing cells. Despite its efficacy in cancer treatment, chemotherapy remains
Department of Psychology, Chung Shan Medical University, No I I0, Section I, Jianguo North Road, Taichung City 4020I, Taiwan

Tel +886424730022 (ext 12324)

Email whwang@csmu.edu.tw
Neuropsychiatric Disease and Treatment 20|9:15 753-76|

753

Dovepress f 5 in 0

http:

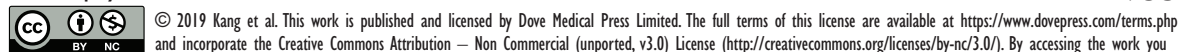

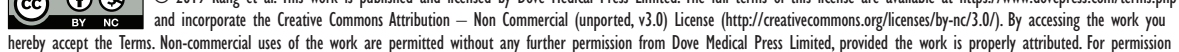
for commercial use of this workh, pleases see paragraphs 4.2 and 5 of our Terms (https://www.dovepress.com/terms.php.). 
associated with a variety of adverse effects, including nausea, vomiting, and fatigue. ${ }^{2}$ Moreover, treatment-related neurocognitive dysfunction in patients with lung cancer has become a matter of heightened concern. ${ }^{2-5}$ A number of studies on small-cell lung cancer patients have demonstrated that diminished verbal fluency, verbal memory, visuospatial ability, and executive function are frequently found before or after chemotherapy. ${ }^{6-8}$

Although non-small-cell lung cancer (NSCLC) accounts for $85 \%$ of all lung cancers, ${ }^{9}$ only a handful of studies have examined the impact of cancer treatment on neurocognitive function in NSCLC patients. Kaasa et al (1988) found that mild cognitive decline was more closely associated with chemotherapy patients than with patients in the radiotherapy group. ${ }^{10}$ Whitney et al (2008) observed that $>60 \%$ of NSCLC patients exhibited noticeable cognitive impairments both before and 1 month after chemotherapy. ${ }^{11}$ Simó et al (2015) demonstrated that NSCLC patients performed significantly worse in verbal memory before chemotherapy than patients with small-cell lung cancer or healthy controls. ${ }^{8}$ These findings suggest that the neurotoxic effect of chemotherapy medications may be one possible mechanism underlying cognitive changes.

Instead of extensively destroying cells, a form of molecular medicine known as targeted therapy has been developed. Targeted therapy works by specifically blocking certain aspects of signaling pathways associated with carcinogenesis and tumor growth. ${ }^{12}$ It should thus be able to reduce treatmentrelated neurotoxicities and adverse effects in patients with lung cancer. However, it remains unclear whether targeted therapy is less likely than conventional chemotherapy to induce neurocognitive deficits in patients with NSCLC.

Both objective and subjective measures are necessary because each provides information relevant to the functioning of cancer patients. ${ }^{13}$ However, it has been well documented that the complications of psychiatric disturbances (especially anxiety and depression) significantly affect perceived cognitive impairments in patients with lung cancer, ${ }^{13-16}$ and this should be appropriately addressed when assessment is performed. In view of the aforementioned matters, this cross-sectional study was designed to compare objective and perceived cognitive performance as well as psychiatric disturbances among patients with NSCLC who were either previously untreated or treated with chemotherapy or targeted therapy.

\section{Patients and methods Subjects}

The study protocol was conducted in accordance with the principles of the Declaration of Helsinki and was approved by the institutional ethics committee of Chiayi Chang Gung Memorial Hospital (approval number CGMH 105-2485C), one of the largest medical centers in southern Taiwan. Written informed consent was obtained upon enrollment from all participants, who were recruited from the CCGMH's outpatient departments of hematology and oncology and pulmonary medicine. Patients were eligible if they had a histologically proven diagnosis of NSCLC, whereas those with any organic brain diseases, a history of mental retardation, psychiatric disorders, premenopausal status, medication affecting the central nervous system, or age older than 80 years were excluded. Moreover, to obtain the greatest possible number of samples, we enrolled NSCLC patients of all stages (I-IV), except for those with metastases. A total of 113 patients with NSCLC were thus enrolled and then classified into three groups based on their cancer treatment type. The untreated control and chemotherapy groups each contained 40 patients, and the targeted therapy group 33 patients. On average, the participants completed all neuropsychological evaluations either within 6 months after diagnosis (for the untreated group) or about 18 months after treatment.

\section{Objective cognitive assessment}

The Chinese versions of tests of each domain were translated and adapted for the Taiwanese population by testing normal subjects. The Mini-Mental State Examination (MMSE) was employed to assess global cognitive function, including orientation, attention, memory, and language. ${ }^{17,18}$ In addition, selected subtests - Vocabulary, Digit Span, and Digit Symbol Substitution - of the Mandarin version of the Wechsler Adult Intelligence Scale-III were used to measure language, working memory, and psychomotor speed, respectively. ${ }^{19}$

Executive function was evaluated using the Color Trails Test, ${ }^{20,21}$ which comprises two parts. The first contains numbers only and evaluates the tracking ability, whereas in the second part, participants must switch between numbers and colors to assess their executive function through cognitive flexibility. The number of errors (irrespective of type) and the completion time (in seconds) were both recorded. Please see Supplementary material S1 outlining the details of the aforesaid cognitive tests.

\section{Subjective cognitive measure}

Subjective cognitive functioning was assessed using the Functional Assessment of Cancer Therapy-Cognitive Function (FACT-cog) scale. ${ }^{22}$ The scale consists of 37 items, with overall cognitive function as the sum of four subscales, namely, perceived cognitive impairments, impact on quality of life, comments from others, and perceived cognitive abilities. 
Higher scores indicate superior cognitive function (ie, lower subjective cognitive impairment).

\section{Psychological assessment}

The Hospital Anxiety and Depression Scale (HADS) was used to assess the severity of depression and anxiety symptoms. ${ }^{23}$ It is divided into two seven-item subscales, one of which specifically focuses on anxiety (HADS-A) and the other on depression (HADS-D). This scale has been widely used in clinical settings and psycho-oncological investigations. Participants with score $\geq 8$ were considered as suffering from HADS-defined anxiety and depression disorder.

\section{Statistical analysis}

All statistical analyses were performed using SPSS version 19. One-way analysis of variance was used to compare total FACT-cog scores and demographic data among the three groups. Analysis of neuropsychological test results included descriptive statistics, namely, means, SDs, frequencies, and percentages. An individual neuropsychological test was regarded as indicating abnormality if the result's SD was $\geq 1.65$ below the normative mean (ie, below the fifth percentile of normative samples). ${ }^{24}$ Similarly, overall performance was defined as "impaired" if any of the test scores were abnormal. The chi-squared $\left(\chi^{2}\right)$ test was used to determine group differences in the proportion of patients with abnormal cognitive performance. Finally, the relationship between cognitive tests and psychiatric measures was assessed using a two-tailed Pearson correlation analysis. $P$-value $<0.05$ was considered to indicate statistical significance.

\section{Results}

\section{Demographic data}

As shown in Table 1, one-way multivariate analysis of variance indicated no significant differences in age and education among the three groups. However, group differences were found for disease duration $\left(\mathrm{F}_{(2,110)}=11.28, P<0.001\right)$. Post hoc comparisons performed using Fisher's least significant difference test indicated that the chemotherapy group's mean years of disease duration were significantly longer than those of the untreated $(P<0.001)$ and targeted therapy
$(P=0.013)$ groups. Moreover, disease duration in the targeted therapy group was significantly longer than in the untreated group $(P=0.049)$.

\section{Neuropsychological test results}

The results of individual neuropsychological tests are detailed in Tables 2 and 3. As seen in Table 3, 35\% of untreated NSCLC patients had impaired performance in at least one cognitive domain, with a comparable proportion found in the chemotherapy (35\%) and targeted therapy (30\%) groups. The proportion of patients with impaired psychomotor speed was the highest (10\%-15\%) across the various cognitive domains. Moreover, a significant proportion of NSCLC patients (15\%-20\%) had borderline or higher scores for depression or anxiety. However, no significant group differences were found for either perceived cognitive performance or the proportion of patients with abnormal objective cognitive performance.

\section{Associations between cognitive performance and psychiatric disturbances} As shown in Table 4, significant correlations were found between total FACT-cog scores and the HADS-A subscale across the three groups. Except for in the targeted therapy group, the HADS-D subscale was also significantly associated with total FACT-cog scores. However, no significant correlations were noted between the majority of objective cognitive tests and the HADS-A and HADS-D subscales. Similarly, there was no significant correlation between total FACT-cog scores and the majority of the objective cognitive tests.

\section{Discussion}

This prospective, cross-sectional pilot study was intended to compare the effects of chemotherapy and targeted therapy on neuropsychological performance and psychiatric symptoms in patients with NSCLC. To our knowledge, this is the first study to explore such therapies in an NSCLC population.

Overall, this study showed that $35 \%$ of untreated NSCLC patients had impaired performance in at least one cognitive domain, with a comparable proportion $(30 \%-35 \%)$ found in

Table I Characteristics of demographic variables

\begin{tabular}{|c|c|c|c|c|c|}
\hline Years & $\begin{array}{l}\text { Non-treatment } n=40 \\
\text { Mean (SD) }\end{array}$ & $\begin{array}{l}\text { Chemotherapy } \mathrm{n}=\mathbf{4 0} \\
\text { Mean (SD) }\end{array}$ & $\begin{array}{l}\text { Targeted therapy } n=33 \\
\text { Mean (SD) }\end{array}$ & $\boldsymbol{F}$ & $P$-value \\
\hline Age & $59.05(6.17)$ & $58.10(8.64)$ & $57.82(8.20)$ & 0.26 & 0.77 \\
\hline Education & 10.00 & 9.68 (3.19) & $10.09(3.66)$ & 0.16 & 0.86 \\
\hline Disease duration & $0.60(1.72)$ & $1.85(1.21)^{\mathrm{a}}$ & $1.15(1.15)^{\mathrm{b}}$ & $11.28 * * *$ & 0.00 \\
\hline
\end{tabular}

Notes: ${ }^{a}$ Chemotherapy group $>$ non-treatment group and targeted therapy group; ${ }^{\mathrm{b}}$ Targeted therapy group $>$ non-treatment group; $* * * P<0.00$ I. 
Table 2 Neuropsychological performance in patients with different types of cancer treatment

\begin{tabular}{|c|c|c|c|}
\hline & $\begin{array}{l}\text { Non-treatment } n=40 \\
\text { Mean (SD) }\end{array}$ & $\begin{array}{l}\text { Chemotherapy } n=40 \\
\text { Mean (SD) }\end{array}$ & $\begin{array}{l}\text { Targeted therapy } n=33 \\
\text { Mean (SD) }\end{array}$ \\
\hline MMSE & $28.35(1.75)$ & $28.28(2.26)$ & $28.03(2.05)$ \\
\hline Vocabulary & $10.03(1.40)$ & $9.55(1.96)$ & $10.33(2.43)$ \\
\hline Digit Span & $10.23(2.66)$ & $9.40(2.27)$ & $10.03(3.20)$ \\
\hline DSS & $8.78(2.86)$ & $8.70(2.72)$ & $9.30(3.18)$ \\
\hline \multicolumn{4}{|l|}{ CTTI } \\
\hline Completion time & $70.90(26.75)$ & $68.15(18.90)$ & $69.92(22.30)$ \\
\hline Errors & $0.05(0.22)$ & $0.07(0.35)$ & $0.03(0.17)$ \\
\hline \multicolumn{4}{|l|}{ СТT2 } \\
\hline Completion time & 130.12 (40.70) & 125.16 (20.68) & I 29.06 (40.39) \\
\hline Number errors & $0.02(0.16)$ & $0.05(0.24)$ & $0.06(0.24)$ \\
\hline Color errors & $0.25(0.68)$ & $0.32(0.59)$ & $0.24(0.76)$ \\
\hline HADS total & $8.70(8.13)$ & $7.85(8.57)$ & $7.06(7.64)$ \\
\hline Anxiety & $4.75(4.62)$ & $3.45(4.84)$ & $3.18(3.88)$ \\
\hline Depression & 3.95 (3.99) & $4.40(4.31)$ & $3.88(4.33)$ \\
\hline
\end{tabular}

Abbreviations: CTT, Color Trails Test; DSS, Digit Symbol Substitution; HADS, Hospital Anxiety and Depression Scale; MMSE, Mini-Mental State Examination.

post-chemotherapy groups. Despite the lower proportion of patients with abnormal cognitive performance, our results are similar to those of another study that found cognitive impairments in $>60 \%$ of NSCLC patients both before and 1 month after chemotherapy. ${ }^{11}$ Differences in the evaluation of cognitive domains and selection of cognitive tests may account for this discrepancy.
Regardless of the nonsignificant group differences, the proportion of patients with impaired psychomotor speed was the highest $(10 \%-15 \%)$ across the various cognitive domains. Several lesion and neuroimaging studies have demonstrated that impaired psychomotor speed is strongly associated with white matter damage. ${ }^{25-27}$ Accordingly, NSCLC patients with psychomotor speed impairment may

Table 3 Comparison of the three groups for various measures according to either mean score or proportion of patients with abnormal test performance

\begin{tabular}{|c|c|c|c|c|c|}
\hline & $\begin{array}{l}\text { Non-treatment } \\
\text { n (\%) } \\
\text { Mean (SD)* }\end{array}$ & $\begin{array}{l}\text { Chemotherapy } \\
\text { n (\%) } \\
\text { Mean (SD)* }\end{array}$ & $\begin{array}{l}\text { Targeted therapy } \\
\mathrm{n}(\%) \\
\text { Mean (SD)* }\end{array}$ & $\chi^{2}$ or $\mathrm{F}$ & $P$-value \\
\hline MMSE & 0 & $2(1.8)$ & $2(1.8)$ & 2.332 & 0.312 \\
\hline Vocabulary & 0 & 0 & 0 & Nil & Nil \\
\hline DSS & $6(15)$ & $4(10)$ & $5(15.2)$ & 0.577 & 0.749 \\
\hline Digit Span & 0 & $2(5)$ & I (3) & 1.960 & 0.375 \\
\hline \multicolumn{6}{|l|}{ CTTI } \\
\hline Completion time & $5(12.5)$ & $2(5)$ & $4(12.12)$ & 1.582 & 0.453 \\
\hline Errors & $2(5)$ & $2(5)$ & I (3.03) & 0.214 & 0.898 \\
\hline \multicolumn{6}{|l|}{ СТT2 } \\
\hline Completion time & $2(5.4)$ & I (2.5) & $3(9.09)$ & 1.523 & 0.467 \\
\hline Number errors & I (2.5) & $3(7.5)$ & $2(6.06)$ & 1.047 & 0.593 \\
\hline Color errors & $3(7.5)$ & $3(7.5)$ & $2(6.06)$ & 0.074 & 0.964 \\
\hline Overall performance & $14(35)$ & $14(35)$ & $10(30)$ & 0.235 & 0.889 \\
\hline HADS-A & $8(20)$ & $6(15)$ & $6(18)$ & 0.351 & 0.839 \\
\hline HADS-D & $8(20)$ & $6(15)$ & $5(15)$ & 0.450 & 0.799 \\
\hline \multicolumn{6}{|l|}{ FACT-cog } \\
\hline Total score & I I 8.88 (9.75) & II 3.90 (14.07) & I I 3.94 (I6.25) & 1.757 & 0.177 \\
\hline $\mathrm{PCl}$ & $66.80(6.4 I)$ & $64.28(8.84)$ & $64.27(8.57)$ & 1.296 & 0.278 \\
\hline CFO & $15.63(1.00)$ & $15.43(1.68)$ & I5.64 (I.58) & 0.262 & 0.770 \\
\hline PCA & $21.13(3.4 I)$ & $19.33(3.68)$ & $19.91(4.90)$ & 2.108 & 0.126 \\
\hline QOL & I5.33 (I.86) & I4.88 (2.54) & $14.12(3.90)$ & 1.666 & 0.194 \\
\hline
\end{tabular}

Note: *the results of the FACT-cog are presented in Mean (SD).

Abbreviations: CFO, comments from others; CTT, Color Trails Test; DSS, Digit Symbol Substitution; FACT-cog, Functional Assessment of Cancer Therapy-Cognitive Function; HADS-A, Hospital Anxiety and Depression Scale-Anxiety; HADS-D, Hospital Anxiety and Depression Scale-Depression; PCA, perceived cognitive abilities; $\mathrm{PCl}$, perceived cognitive impairments; QOL, quality of life; MMSE, Mini-Mental State Examination. 


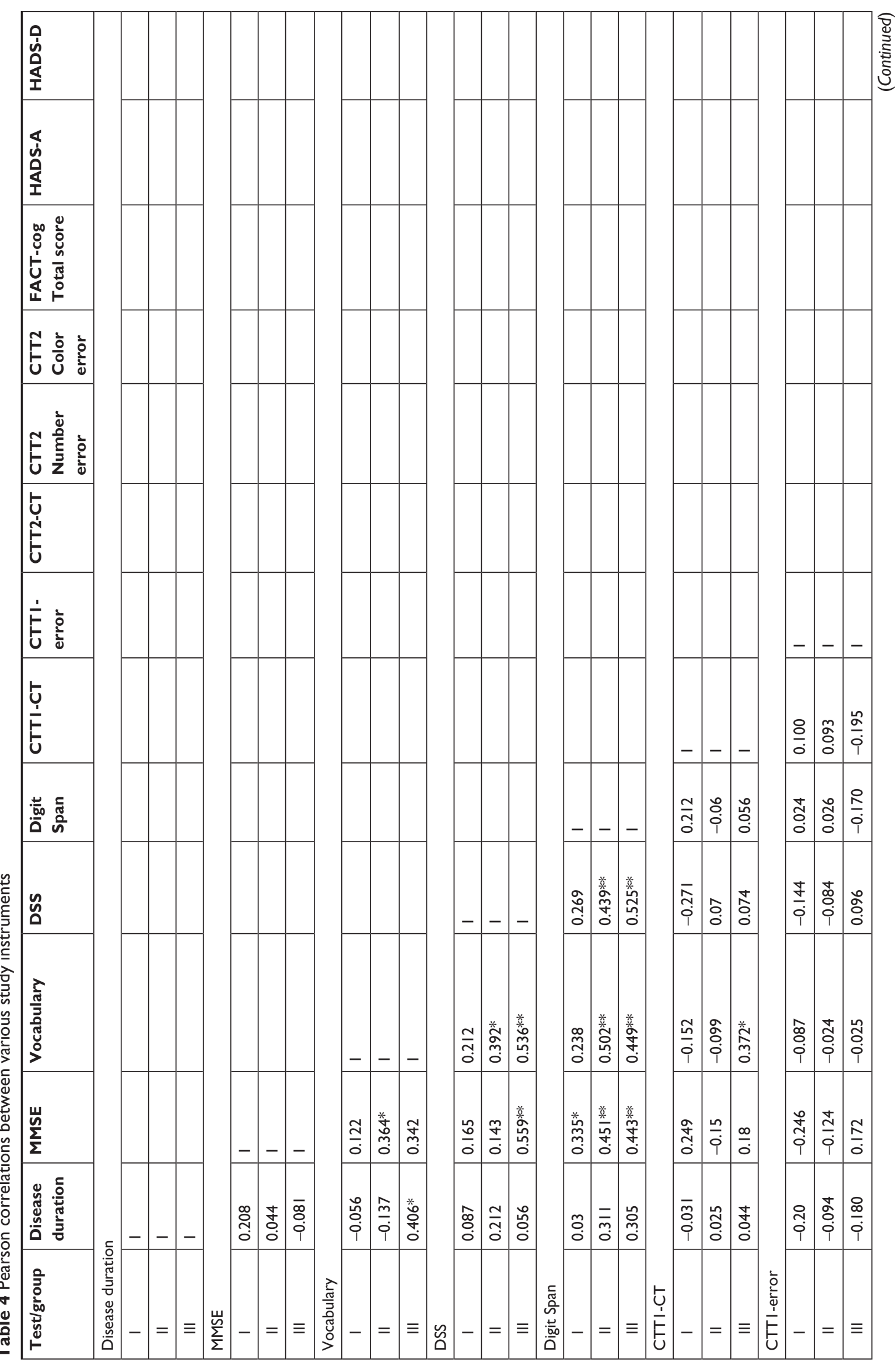




\begin{tabular}{|c|c|c|c|c|c|c|c|c|c|c|c|c|c|c|c|c|c|c|c|c|c|c|c|c|c|c|c|}
\hline $\begin{array}{l}0 \\
\dot{\omega} \\
\stackrel{0}{\alpha} \\
\text { I }\end{array}$ & & & & & & & & & & & & & & & & & & & & & & & & - & - & - & 文 \\
\hline 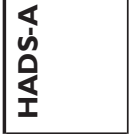 & & & & & & & & & & & & & & & & & & & & - & - & - & & 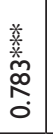 & 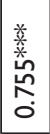 & 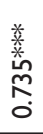 & \\
\hline 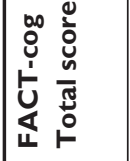 & & & & & & & & & & & & & & & & - & - & - & & 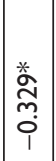 & 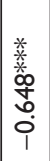 & 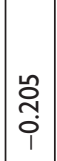 & & 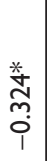 & 䔡 & $\begin{array}{l}\text { *. } \\
\text { o } \\
\text { o. } \\
\text { i }\end{array}$ & \\
\hline$E$ E & & & & & & & & & & & & - & - & - & & $\begin{array}{c}\text { sô. } \\
0 \\
0 \\
i\end{array}$ & $\frac{a}{m}$ & $\begin{array}{l}n \\
0 \\
0 \\
i\end{array}$ & & $\frac{\hat{0}}{\bar{i}}$ & $\frac{2}{9}$ & 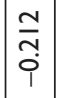 & & $\frac{\text { Cे }}{i}$ & 辛 & $\frac{d}{\frac{1}{2}}$ & \\
\hline 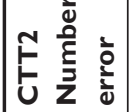 & & & & & & & -1 & - & - & & & 8 & $\begin{array}{l}\stackrel{2}{0} \\
0 \\
0\end{array}$ & $\begin{array}{l}\stackrel{*}{*} \\
\stackrel{\sigma}{\sigma} \\
\stackrel{0}{0}\end{array}$ & & $\stackrel{\grave{T}}{\grave{O}}$ & 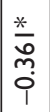 & $\begin{array}{l}2 \\
2 \\
0 \\
0 \\
i\end{array}$ & & 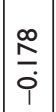 & 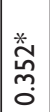 & $\frac{\tilde{a}}{\bar{i}}$ & & \begin{tabular}{l} 
ț \\
\multirow{1}{*}{} \\
i
\end{tabular} & $\frac{\infty}{\frac{\infty}{0}}$ & $\frac{\text { to }}{\text { i }}$ & \\
\hline U & & - & - & - & & & $\begin{array}{l}\tilde{0} \\
0 \\
0 \\
i\end{array}$ & $\begin{array}{l}0 \\
0 \\
0 \\
i\end{array}$ & 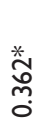 & & & 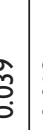 & $\begin{array}{l}\sigma \\
\partial \\
O\end{array}$ & 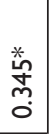 & & 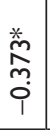 & స్ & ồ. & & $\begin{array}{l}\tilde{\Xi} \\
0 \\
0\end{array}$ & $\frac{1}{\frac{\pi}{0}}$ & 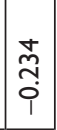 & & $\begin{array}{l}\infty \\
\stackrel{0}{0} \\
0 \\
0\end{array}$ & $\frac{a}{a}$ & $\frac{\infty}{i}$ & \\
\hline 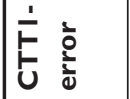 & & $\frac{n}{0}$ & $\begin{array}{l}0 \\
\vdots \\
0 \\
0 \\
i\end{array}$ & 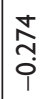 & & & $\begin{array}{l}\infty \\
0 \\
0 \\
i\end{array}$ & $\begin{array}{l}\infty \\
0 \\
0 \\
i \\
i\end{array}$ & $\begin{array}{l}\text { o } \\
\text { Oó } \\
\text { i }\end{array}$ & & 辛 & . & $\begin{array}{l}0 \\
\text { ò } \\
\text { i }\end{array}$ & $\begin{array}{l}\frac{2}{0} \\
0 \\
i \\
i\end{array}$ & & $\begin{array}{c}\tilde{\sigma} \\
\delta \\
0 \\
i\end{array}$ & $\begin{array}{l}\infty \\
\tilde{O} \\
0 \\
0\end{array}$ & $\frac{2}{0}$ & & $\frac{\infty}{i}$ & $\begin{array}{l}\bar{\alpha} \\
0 \\
0 \\
i\end{array}$ & $\frac{\overline{0}}{\bar{i}}$ & & $\begin{array}{l} \pm \\
\dot{0} \\
\end{array}$ & 苂 & $\frac{\overline{0}}{i}$ & \\
\hline È & & 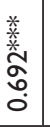 & 釈 & 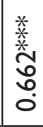 & & & $\begin{array}{l}\frac{n}{2} \\
\\
\end{array}$ & $\begin{array}{c}\frac{1}{m} \\
\dot{0} \\
i\end{array}$ & ণ্ণ & & & Sُ & 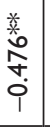 & 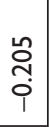 & & 茎 & $\begin{array}{l}n \\
\text { o } \\
0 \\
0\end{array}$ & $\begin{array}{l}m \\
\hat{0} \\
0 \\
0\end{array}$ & & $\begin{array}{l}2 \\
\hat{\sigma} \\
0 \\
0\end{array}$ & 负 & 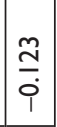 & & 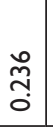 & $\frac{a}{\frac{m}{0}}$ & $\begin{array}{l}\text { \& } \\
\text { i } \\
\text { i }\end{array}$ & के \\
\hline 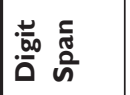 & & $\begin{array}{l}\tilde{\tilde{o}} \\
0 \\
0\end{array}$ & $\stackrel{\infty}{\frac{\infty}{\infty}}$ & 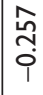 & & & $\overline{\bar{O}}$ & $\frac{\frac{2}{\sigma}}{\frac{i}{i}}$ & $\frac{\mathbb{N}}{0}$ & & & $\begin{array}{l}n \\
\frac{n}{1} \\
1\end{array}$ & $\begin{array}{l}0 \\
\hat{0} \\
0\end{array}$ & 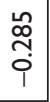 & & 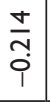 & $\begin{array}{l}\bar{\alpha} \\
0 \\
i \\
\end{array}$ & $\begin{array}{l}* \\
0 \\
0 \\
i \\
\end{array}$ & & $\begin{array}{c}\frac{*}{o} \\
\frac{m}{0} \\
\end{array}$ & $\begin{array}{l}0 \\
0 \\
0 \\
0 \\
i\end{array}$ & 芯 & & $\frac{\stackrel{*}{*}}{m}$ & $\frac{\hat{m}}{i}$ & $\frac{\infty}{\infty}$ & \\
\hline ڤ̆ & & $\begin{array}{l}\text { స్ } \\
\text { ஸे } \\
\end{array}$ & $\begin{array}{l}0 \\
\tilde{\alpha} \\
0 \\
i \\
i\end{array}$ & $\begin{array}{l}\infty \\
0 \\
0 \\
0 \\
i\end{array}$ & & & $\frac{\hat{m}}{0}$ & $\frac{\overline{0}}{0}$ & $\begin{array}{l}\text { ?े } \\
0 \\
0 \\
\end{array}$ & & 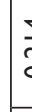 & $\frac{d}{d}$ & $\begin{array}{l}\tilde{\tilde{o}} \\
0 \\
0\end{array}$ & $\frac{\hat{\infty}}{i}$ & & 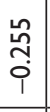 & $\frac{i}{i}$ & $\begin{array}{l}\overline{\mathbf{o}} \\
0 \\
\mathbf{i} \\
\end{array}$ & & $\begin{array}{l}\bar{\infty} \\
0 \\
0\end{array}$ & $\begin{array}{l}\bar{a} \\
\text { ò } \\
\end{array}$ & $\overline{0}$ & & 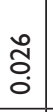 & 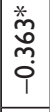 & $\begin{array}{l}\text { ळ } \\
\text { స్ }\end{array}$ & \\
\hline 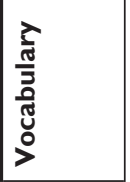 & & 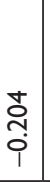 & $\frac{n}{\overline{0}}$ & 今̃ & & & $\begin{array}{l}\hat{8} \\
0 \\
1\end{array}$ & $\frac{g}{\sigma}$ & $\overline{\bar{~}}$ & & & $\frac{\infty}{5}$ & 훙 & $\begin{array}{c}8 \\
\tilde{m} \\
i \\
i\end{array}$ & & $\begin{array}{l}\stackrel{n}{2} \\
\hat{O} \\
0\end{array}$ & $\frac{\pi}{0}$ & $\begin{array}{l}\overline{\hat{a}} \\
0 \\
\mathbf{i} \\
\end{array}$ & & તે & $\frac{\pi}{0}$ & 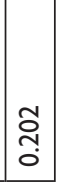 & & $\begin{array}{c}\frac{*}{0} \\
\stackrel{m}{0} \\
0\end{array}$ & $\begin{array}{l}n \\
\hat{0} \\
0 \\
i\end{array}$ & $\frac{\bar{\sigma}}{\sigma}$ & \\
\hline$\sum_{\Sigma}^{\frac{\omega}{\Sigma}}$ & & $\begin{array}{l}0 \\
0 \\
0 \\
0\end{array}$ & 柋 & $\frac{o}{0}$ & & & $\begin{array}{l}\tilde{m} \\
\tilde{o} \\
0\end{array}$ & $\begin{array}{l}\overline{0} \\
0 \\
\end{array}$ & $\frac{n}{\circ}$ & & (x) & to & $\begin{array}{l}\hat{\Delta} \\
0 \\
\dot{1}\end{array}$ & $\frac{\sigma}{i}$ & & 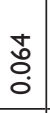 & 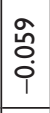 & $\begin{array}{c}n \\
\vdots \\
0 \\
i \\
\end{array}$ & & $\begin{array}{l}0 \\
0 \\
0 \\
i \\
\end{array}$ & $\frac{\bar{m}}{\bar{i}}$ & $\frac{2}{0}$ & & $\stackrel{\infty}{\underset{0}{0}}$ & 产 & 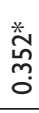 & \\
\hline 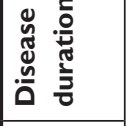 & & $\frac{1}{0}$ & $\frac{i}{i}$ & 。े & & & $\frac{2}{0}$ & 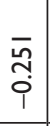 & $\begin{array}{l}\text { సे } \\
\text { ठे }\end{array}$ & & & ?. & 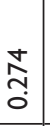 & $\begin{array}{l}\infty \\
0 \\
0 \\
0\end{array}$ & & $\begin{array}{l}\stackrel{+}{\mathrm{N}} \\
\text { Oे }\end{array}$ & 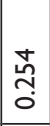 & $\overline{\tilde{o}}$ & & \begin{tabular}{l}
\multirow{N}{N}{} \\
$\stackrel{i}{i}$
\end{tabular} & 莣 & $\begin{array}{l}\hat{n} \\
0 \\
0\end{array}$ & & $\frac{2}{i}$ & 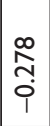 & $\begin{array}{l}\overline{\tilde{o}} \\
\dot{i}\end{array}$ & \\
\hline 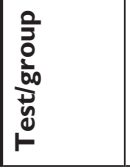 & $\begin{array}{c}\llcorner \\
\dot{U} \\
\dot{E}\end{array}$ & - & $=$ & $\equiv$ & & $\begin{array}{l}\frac{1}{0} \\
\frac{2}{0} \\
0 \\
\frac{0}{0} \\
\\
ن\end{array}$ & - & $=$ & $\equiv$ & & & & $=$ & $\equiv$ & 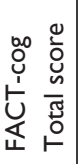 & - & $=$ & $\equiv$ & 晏 & - & $=$ & $\equiv$ & $\mid \begin{array}{l}0 \\
\text { 晏 } \\
\text { 全 }\end{array}$ & - & $=$ & $\equiv$ & \\
\hline
\end{tabular}


also exhibit white matter lesions. This assumption is partly supported by Simó et al (2015), who investigated cognitive and brain structure changes in patients with lung cancer and found that NSCLC patients exhibited verbal memory deficits and widespread white matter damage, compared with healthy controls. ${ }^{8}$ Due to clinical time constraints and patient fatigue, we employed the MMSE to assess various domains of cognitive function, including verbal memory. However, it has been suggested that the MMSE lacks sensitivity to very mild cognitive impairments. ${ }^{28}$ A short but comprehensive neuropsychological battery focusing specifically on certain cognitive domains is thus recommended for future research.

The present study also found no significant differences among the three groups in the proportion of patients with abnormal performance in both objective and subjective cognitive tests. Whitney et al (2008) found that NSCLC patients exhibited cognitive declines at a 1-month follow-up, but those problems apparently dissipated by 7 months of posttreatment, ${ }^{11}$ thus suggesting that cognitive declines during cancer treatment were only temporary. The mean disease duration of each therapy group in our study was $>12$ months, which may explain why chemotherapies did not significantly increase the number of patients with cognitive impairments relative to the untreated group. Furthermore, the proportion of patients with abnormal cognitive performance did not significantly differ between the chemotherapy and targeted therapy groups, although the latter tended toward a lower number. A longitudinal study exploring the effects of chemotherapy and targeted therapy on cognitive function is warranted.

Consistent with other studies using HADS, ${ }^{29,30}$ our results indicated that a significant proportion of NSCLC patients (15\%-20\%) exhibited borderline or even higher scores for depression or anxiety, which were also significantly associated with subjective cognitive performance. Moreover, we found that objective and perceived cognitive performances were unrelated. These findings support the observation of Hutchinson et al (2012) that perceived impairment may be an indicator of psychological distress rather than cognitive impairment. ${ }^{13}$ In summary, the clinical implication of these findings is that both treated and untreated patients with lung cancer may suffer from psychological disturbances. Therefore, clinicians should pay greater attention to patients who are susceptible to depression and provide prompt treatment to improve their cognitive performance.

There are several limitations to our study. First, given its cross-sectional design, this study could not determine the impact of both cancer and cancer treatments on cognitive function over time. Second, because patients' treatment histories were not considered in this study, we could not entirely exclude the potential influence of other chemotherapy medications on patients' performance. A longitudinal study is required to address this question. Finally, this study did not assess and match participants closely for intelligence level, which could confound patients' performance on neuropsychological tests. Therefore, consideration of this factor in future research is warranted.

\section{Conclusion}

Our study demonstrated that 1) a substantial proportion of NSCLC patients exhibited cognitive impairments (especially regarding psychomotor speed) and psychiatric disturbances; 2) there were no significant differences between the three patient groups for any subjective and objective measure of cognitive deficit; and 3) perceived cognitive impairment was significantly associated with depression and anxiety. Prompt treatment of psychiatric disorders to minimize their impact is therefore recommended.

\section{Acknowledgments}

This study was supported by grants from Chang Gung Memorial Hospital, Chiayi, Taiwan (grant number: CMRPG6F0321, CORPG6G0101, CORPG6G0141). The funder had no role in the design and conduct of the study; collection, management, analysis, and interpretation of the data; preparation, review, or approval of the manuscript; and decision to submit the manuscript for publication.

\section{Author contributions}

All the authors contributed to data analysis, drafting, and critical revision of this paper, approved the final version for publication and agreed to be accountable for all aspects of the research.

\section{Disclosure}

The authors report no conflicts of interest in this work.

\section{References}

1. Ministry of Health and Welfare of the Republic of China (2017). Statistics of Causes of Death. Available from: from http://www.mohw.gov.tw/ np-128-2.html. Accessed September 12, 2018.

2. Wefel JS, Kayl AE, Meyers CA. Neuropsychological dysfunction associated with cancer and cancer therapies: a conceptual review of an emerging target. Br J Cancer. 2004;90(9):1691-1696.

3. Asher A, Myers JS. The effect of cancer treatment on cognitive function. Clin Adv Hematol Oncol. 2015;13(7):441-450.

4. Jansen CE, Miaskowski C, Dodd M, Dowling G, Kramer J. A metaanalysis of studies of the effects of cancer chemotherapy on various domains of cognitive function. Cancer. 2005;104(10):2222-223315.

5. Pendergrass JC, Targum SD, Harrison JE. Cognitive impairment associated with cancer: a brief review. Innov Clin Neurosci. 2018;15(1-2):36-44. 
6. Meyers CA, Byrne KS, Komaki R. Cognitive deficits in patients with small cell lung cancer before and after chemotherapy. Lung Cancer. 1995; 12(3):231-235.

7. Grosshans DR, Meyers CA, Allen PK, Davenport SD, Komaki R. Neurocognitive function in patients with small cell lung cancer: effect of prophylactic cranial irradiation. Cancer. 2008;112(3):589-595.

8. Simó M, Root JC, Vaquero L, et al. Cognitive and brain structural changes in a lung cancer population. J Thoracic Oncol. 2015;10(1):38-45.

9. Molina JR, Yang P, Cassivi SD, Schild SE, Adjei AA. Non-small cell lung cancer: epidemiology, risk factors, treatment, and survivorship. Mayo Clin Proc. 2008;83(5):584-594.

10. Kaasa S, Olsnes BT, Mastekaasa A. Neuropsychological evaluation of patients with inoperable non-small cell lung cancer treated with combination chemotherapy or radiotherapy. Acta Oncol. 1988;27(3):241-246.

11. Whitney KA, Lysaker PH, Steiner AR, et al. Is "chemobrain" a transient state? A prospective pilot study among persons with non-small cell lung cancer. J Support Oncol. 2008;6(7):313-321.

12. Minguet J, Smith KH, Bramlage P. Targeted therapies for treatment of non-small cell lung cancer - Recent advances and future perspectives. Int J Cancer. 2016;138(11):2549-2561.

13. Hutchinson AD, Hosking JR, Kichenadasse G, Mattiske JK, Wilson C. Objective and subjective cognitive impairment following chemotherapy for cancer: a systematic review. Cancer Treat Rev. 2012;38(7):926-934.

14. Shi Y, Gu F, Hou L-L, Hu Y-Q, Yq H. Self-reported depression among patients with non-small cell lung cancer. Thorac Cancer. 2015; 6(3):334-337.

15. Tang P-L, Wang H-H, Chou F-H. A systematic review and metaanalysis of demoralization and depression in patients with cancer. Psychosomatics. 2015;56(6):634-643.

16. Steinberg T, Roseman M, Kasymjanova G, et al. Prevalence of emotional distress in newly diagnosed lung cancer patients. Support Care Cancer. 2009;17(12):1493-1497.

17. Folstein MF, Folstein SE, Mchugh PR. "Mini-mental state". A practical method for grading the cognitive state of patients for the clinician. J Psychiatr Res. 1975;12(3):189-198.

18. Shyu YI, Yip PK. Factor structure and explanatory variables of the Mini-Mental State Examination (MMSE) for elderly persons in Taiwan. J Formos Med Assoc. 2001;100:676-683.
19. Chen JH, Chen HY. Wechsler Adult Intelligence Scale. 3rd ed. Taipei: Chinese Behavioral Science Corp; 2002 [Manual].

20. D'Elia L, Satz P, Uchiyama C, White T. Color Trails Test Professional Manual. Odessa, FL: Psychological Assessment Resources; 1996.

21. Kuo HY, Hua MS. Color Trails Test. Taipei: Chinese Behavioral Science Corp; 2002 [Manual].

22. Wagner L, Sweet J, Butt Z, et al. Measuring patient self-reported cognitive function: development of the functional assessment of cancer therapy-cognitive function instrument. J Support Oncol. 2009;7(6): W32-W39.

23. Zigmond AS, Snaith RP. The hospital anxiety and depression scale. Acta Psychiatr Scand. 1983;67(6):361-370.

24. Lezak MD, Howieson DB, Bigler ED, Tranel D, Neuropsychological Assessment. New York. 5th ed. US: Oxford University Press; 2012.

25. Bolandzadeh N, Davis JC, Tam R, Handy TC, Liu-Ambrose T. The association between cognitive function and white matter lesion location in older adults: a systematic review. BMC Neurol. 2012;12(126):126.

26. Lam CLM, Yiend J, Lee TMC. Imaging and neuropsychological correlates of white matter lesions in different subtypes of mild cognitive impairment: a systematic review. NeuroRehabilitation. 2017; 41(1):189-204

27. Hyett MP, Perry A, Breakspear M, Wen W, Parker GB. White matter alterations in the internal capsule and psychomotor impairment in melancholic depression. PLoS One. 2018;13(4):e0195672.

28. Lindner OC, Phillips B, Mccabe MG, et al. A meta-analysis of cognitive impairment following adult cancer chemotherapy. Neuropsychology. 2014;28(5):726-740.

29. Chabowski M, Polański J, Jankowska-Polańska B, Janczak D, Rosińczuk J. Is nutritional status associated with the level of anxiety, depression and pain in patients with lung cancer? J Thorac Dis. 2018; 10(4):2303-2310.

30. Schellekens MPJ, van den Hurk DGM, Prins JB, et al. The suitability of the hospital anxiety and depression scale, distress thermometer and other instruments to screen for psychiatric disorders in both lung cancer patients and their partners. J Affect Disord. 2016;203:176-183. 


\section{Supplementary materials Supplementary SI Description of cognitive tests}

The Vocabulary subtest of the Wechsler Adult Intelligence Scale-III (WAIS-III):

It comprises 33 words that are listed in order of difficulty. The participants are asked for definitions of words. One or two points are given for each acceptable definition, depending on its accuracy and aptness.

The Digit Span subtest of the WAIS-III:

It comprises two different tests, Digits Forward and Digits Backward, each of which involves different mental activities. Both tests consist of pairs of random numbers of increasing sequence length that the examiner reads aloud. The number of correct repetitions is measured.

The Digit Symbol Substitution subtest of the WAIS-III:

It consists of digit-symbol pairs followed by a list of digits. Under each digit, the participants should write down the corresponding symbol as fast as possible. The number of correct symbols within the allowed time (120 seconds) is measured.

The Color Trails Test (CTT):

It comprises two parts, each involving numbered circles that are printed with vivid pink or yellow backgrounds. For the CTT Part 1, the participants use a pencil to rapidly connect circles numbered 1 through 25 in sequence. For the CTT Part 2, the participants rapidly connect numbered circles in sequence, but alternates between pink and yellow colors. The length of time (in seconds) to complete each trial is recorded, along with qualitative features of performance indicative of brain dysfunction, such as near-misses, prompts, number sequence errors, and color sequence errors. ${ }^{1}$

\section{Reference}

1. D'Elia L, Satz P, Uchiyama C, White T. Color Trails Test Professional Manual. Odessa, FL: Psychological Assessment Resources; 1996.
Neuropsychiatric Disease and Treatment

\section{Publish your work in this journal}

Neuropsychiatric Disease and Treatment is an international, peerreviewed journal of clinical therapeutics and pharmacology focusing on concise rapid reporting of clinical or pre-clinical studies on a range of neuropsychiatric and neurological disorders. This journa is indexed on PubMed Central, the 'PsycINFO' database and CAS

\section{Dovepress}

and is the official journal of The International Neuropsychiatric Association (INA). The manuscript management system is completely online and includes a very quick and fair peer-review system, which is all easy to use. Visit http://www.dovepress.com/testimonials.php to read real quotes from published authors. 